\title{
Breastfeeding and Coronavirus Disease 2019 (COVID-19) Vaccination: Position Statement of Indian Academy of Pediatrics Advisory Committee on Vaccination and Immunization Practices
}

\author{
Srinivas G Kasi, ${ }^{1}$ Shashi Kant Dhir, ${ }^{2}$ S Shivananda, ${ }^{3}$ Sanjay Marathe, ${ }^{4}$ Kripasindhu ChatterJee, ${ }^{5}$ Sunil \\ Agarwalla, ${ }^{6}$ Sanjay Verma, ${ }^{7}$ Abhay K Shah, ${ }^{8}$ Sanjay Srirampur, ${ }^{9}$ Srinivas Kalyani, ${ }^{10}$ Harish Kumar \\ Pemde, ${ }^{11}$ S Balasubramanian, ${ }^{12}$ GV Basavaraja, ${ }^{13}$ Bakul J Parekh, ${ }^{14}$ Remesh Kumar, ${ }^{15}$ Piyush Gupta ${ }^{16}$ \\ From ${ }^{1}$ Kasi Clinic, Jayanagar, Bengaluru, Karnataka; ${ }^{2}$ Guru Gobind Singh Medical College, Faridkot, Punjab; ${ }^{3}$ Fortis Hospital, \\ Banneraghatta Road, Bengaluru, Karnataka; ${ }^{4}$ Marathe Child Care Hospital, Nagpur, Maharashtra; ${ }^{5}$ Gouri Devi Institute of \\ Medical Science and Hospital, Durgapur, West Bengal; ${ }^{6}$ MKCG MCH, Berhampur, Odisha; ${ }^{7}$ Postgraduate Institute of Medical \\ Education and Research, Chandigarh, ${ }^{8} \mathrm{Dr}$ Abhay K Shah Children Hospital, Ahmedabad, Gujarat, ${ }^{9}$ Aditya Super speciality \\ Hospital, Hyderabad, Telangana; ${ }^{10}$ Niloufer Hospital, Osmania medical College, Hyderabad; ${ }^{11}$ Lady Hardinge Medical College, \\ New Delhi; ${ }^{12}$ Kanchi Kamakoti Childs Trust Hospital, Chennai, Tamil Nadu; ${ }^{13}$ IGICH, Bengaluru, Karnataka; ${ }^{14}$ Bakul Parekh \\ Hospital for Children, Mumbai, Maharashtra; ${ }^{15}$ Apollo Adlux Hospital, Cochin, Kerala; ${ }^{16}$ University College of Medical Sciences, \\ New Delhi. \\ Correspondence to: Srinivas G Kasi, Convener, ACVIP of IAP, Kasi Clinic, 2nd Cross, 3rd Block, Jayanagar, Bengaluru, \\ Karnataka.sgkasi@gmail.com
}

Justification: In India, till recently, breastfeeding women have been excluded from the coronavirus disease (COVID-19) vaccination program, rendering a significant population of the country, including frontline workers, ineligible to derive the benefits of the COVID-19 vaccine rollout. Objective: The objective of this recommendation is production of an evidence-based document to guide the pediatricians to give advice to breastfeeding mothers regarding the safety of COVID-19 vaccines in lactating women. Process: Formulation of key question was done under the chairmanship of president of the IAP. It was followed by review of literature regarding efficacy and safety of COVID-19 vaccines in breastfeeding women. The recommendations of other international and national professional bodies were also deliberated in detail. The available data was discussed in the ACVIP focused WhatsApp group. Opinion of all members was taken and the final document was prepared after achieving consensus. Recommendations: The IAP/ACVIP recommends the administration of COVID-19 vaccines to all breastfeeding women. The IAP/ACVIP endorses the recent recommendation of the Government of India, to consider all breastfeeding women as eligible for COVID-19 vaccination.

Keywords: Lactation, Maternal, Protection, SARS-CoV-2.

$\mathrm{T}$ he development and implementation of coronavirus disease 2019 (COVID-19) vaccination program has been one of the recent and most prominent demonstrations of the power of modern science. As of 14 May, 2021, in India, a total of 13986142 ( 1 dose) and 39784951 (2 doses) have been administered [1,2]. Ever since the initiation of the COVID -19 vaccination program in India, pregnant and breastfeeding women had been excluded from the vaccination program [3]. Recently, the Government of India (GoI) has published a circular, recommending administration of COVID-19 vaccines in breastfeeding women [4]. Failure to include pregnant and lactating women in the phase 3 studies of the mRNA vaccines, Astra-Zeneca vaccine and Covaxin (Bharat Biotech Ltd) and consequent lack of safety data, were the cited reasons for excluding this group. If this cohort of pregnant and breastfeeding mothers continued to be excluded, a significant population of the country, including frontline workers, would have been left unprotected. Hence, the recent recommendation from the $\mathrm{GoI}$ is a welcome step.

\section{OBJECTIVE}

The objective of this recommendation is production of an evidence-based document to guide the pediatricians to give advice to breastfeeding mothers regarding the safety of COVID-19 vaccines in lactating women.

\section{PROCESS}

Formulation of key question was done under the chairmanship of President of the Indian Academy of Pediatrics (IAP). It was followed by review of literature regarding efficacy and safety of COVID-19 vaccines in breastfeeding women. The recommendations of other international and national professional bodies were also deliberated in detail. The available data was discussed in the Advisory Committee on Vaccines and Immunization Practices (ACVIP) focused WhatsApp group. Opinion of all members was taken and the final document was 
prepared after the consensus and was approved by all members of the ACVIP (authors of the guidelines).

\section{BREASTFEEDING AND COVID-19 VACCINES}

The theoretical risk of COVID-19 vaccination in breastfeeding mothers and the potential harm to the infant is unknown. However, it is to be noted that none of the vaccines available for the COVID-19 contains live virus. There is no plausible biological mechanism to explain how an inactivated vaccine, given to the mother, would cause harm to a breastfed baby [5].

The COVID-19 vaccines presently available in India, i.e., Covishield and Covaxin are classified as inactivated (non-live) vaccines. Theoretically, administration of these vaccines to breastfeeding women should not render any harm to the breastfed infant. While the safety of adenovirus vectors in pregnancy and lactation is not established, wild adenoviral infections are present worldwide and have not been associated with teratogenic effects in the fetus or newborns [6]. With the exception of small pox and yellow fever vaccines no other vaccine is contraindicated during breast feeding [7].

Breast milk is a rich source of antibodies for the infant. Milk produced by infected mothers is a source of anti-SARS-CoV-2 IgA and IgG and neutralizes SARSCoV-2 activity [8].

Studies have shown that maternal vaccination with the mRNA vaccine results in high titers of RBD-IgG binding antibodies and neutralizing antibodies as measured by the pseudovirus neutralizing tests (NT50), in maternal serum. High titers of RBD-IgG binding antibodies, neutralizing antibodies as measured by the NT50 and robust T-cell responses, as measured by ELISPOT and intra-cellular cytokine staining, have been demonstrated in the breast milk of mothers vaccinated with the mRNA vaccines [9]. In another study, involving six lactating women who received two doses of SARS-CoV-2 vaccine, significantly elevated levels of SARS-CoV-2 specific IgG and IgA antibodies in breast milk was observed, beginning at Day 7 after the initial vaccine dose, with an IgG-dominant response [10]. These SARS-CoV-2 specific immunoglobulins and products of the T-cell responses in breast milk may be protective for infants.

There is a paucity of data on immunological parameters in breastmilk following the administration of the AstraZeneca COVID-19 vaccine in lactating women. Thus, merely the absence of data should not exclude lactating women from getting the benefits of COVID-19 vaccination.

\section{RECOMMENDATIONS FROM OTHER PROFESSIONAL BODIES}

Many international and national recommending bodies have now recommended administration of COVID-19 vaccines in breastfeeding women (Table I).

While some have recommended the COVID-19 vaccines in breastfeeding women who are in the priority groups, after fully informing them about the benefits and risks of vaccination, some authorities have recommended the vaccine for all breastfeeding women. All have emphasized that breast feeding should be continued after vaccination.

Table I Recommendations for Coronavirus Disease 2019 (COVID-19) Vaccine for Lactating Women by Various National and International Bodies

\begin{tabular}{ll}
\hline Agency & Recommendation \\
\hline World Health Organization [11] & $\begin{array}{l}\text { Developmental and health benefits of breastfeeding should be considered along with the } \\
\text { mother's clinical need for immunization against COVID-19. WHO does not recommend } \\
\text { discontinuing breastfeeding after vaccination. }\end{array}$
\end{tabular}

American College of Obstetricians and Gynecologists [12]

International Federation of Gynecology and Obstetrics [13]

Federation of Obstetric and Gynaecological Societies of India [14]

Australian and New Zealand health authorities $[15,16]$

Italian Scientific Society [17]
COVID-19 vaccines should be offered to lactating individuals similar to non-lactating individuals.

Supports offering COVID-19 vaccination to pregnant and breastfeeding women.

COVID-19 vaccine should extend to pregnant and lactating women. The very real benefits of vaccinating pregnant and lactating women seem to far outweigh any theoretical and remote risks of vaccination.

Recommend COVID-19 vaccines in breast feeding women. They have emphasized that there are no concerns about their safety in breastfeeding women or their babies.

Decision whether or not to administer the COVID-19 vaccine to the breastfeeding woman should be made after mutual agreement between her and the health professionals, considering specific health, social, familiar and work conditions. 


\section{IAP-ACVIP RECOMMENDATIONS}

1. Breastfeeding is very beneficial in the first years of life for nutrition and the protection provided by it against infectious agents. This is of crucial importance in developing countries like India.

2. The benefits of COVID-19 vaccination should not be denied to breastfeeding women as the real benefits are much more than the "theoretical risks."

3. The IAP/ACVIP strongly recommends the administration of COVID-19 vaccines to all breastfeeding women.

4. The IAP/ACVIP endorses the recent decision of the GOI, to consider breastfeeding women eligible for COVID-19 vaccination.

Contributors: All authors were part of the IAP ACVIP team that formulated these guidelines. PG, BJP, GVB, and SGK: conceived the Guidelines, prepared the agenda, and executed administratively. PG and SGK: led the discussions and all the members actively participated. SGK, SKD, SV, HKP, AS reviewed the literature on national and international guidelines, SGK, SM, SKA, SS, SK reviewed the literature on safety of COVID vaccines in breast feeding women, $\mathrm{S}, \mathrm{KC}, \mathrm{SK}$ reviewed the literature on immunological parameters in breast milk. SGK, SKD wrote the first draft. The first draft was peer reviewed by PG, SBS. PG, BJK, PG, GVB, RK provided intellectual inputs and overall guidance at every step. PG, BJP, GVB provided the administrative support from the Indian Academy of Pediatrics and coordinated between the team and executive board members of the Academy. The final document was drafted by SGK and SKD; and edited by PG and SBS. All authors approved the final recommendations of the guidelines.

Funding: None; Competing interests: None stated.

\section{REFERENCES}

1. WHO Coronavirus (COVID-19) Dashboard. Accessed May 15, 2021. Available from: https://COVID19. who.int

2. India: WHO Coronavirus Disease (COVID-19) Dashboard With Vaccination Data. Accessed May 15, 2021. Available from: https:// COVID19.who.int

3. COVID-19 vaccines operational guidelines. Ministry of health and family welfare. Accessed May 15, 2021. Available from: https:// www.mohfw.gov.in/pdf/ COVID19VaccineOG111Chapter16.pdf

4. New Recommendations of NEGVAC accepted by Union Ministry of Health. Accessed May 21, 2021. Available from: https:// pib.gov.in/Press ReleasePage.aspx?PRID $=1719925$

5. Drugs and Lactation Database (LactMed). Bethesda (MD): National Library of Medicine (US); 2006-. COVID-19 vaccines. [Updated 2021 Apr 19]. Accessed May 15, 2021. Available from: https://www.ncbi.nlm.nih.gov/books/NBK565969/

6. EMA, European Medicines Agency. Committee for Medicinal Products for Human Use (CHMP). Assessment Report - COVID19 Vaccine AstraZeneca. EMA/94907/2021, 2021. Assessed January 292021 Available from: https://www.ema.europa.eu/en/documents/assessment-report/COVID-19-vaccine-astrazeneca-eparpublic-assessment-report_en.pdf

7. Sachs HC, Committee On Drugs. The transfer of drugs and therapeutics into human breast milk: an update on selected topics. Pediatrics. 2013;132:e796-809.

8. Pace RM, Williams JE, Järvinen KM, et al. COVID-19 and human milk: SARS-CoV-2, antibodies, and neutralizing capacity. medRxiv [Preprint]. 2020 Sep 18.

9. Collier A-RY, McMahan $\mathrm{K}$, Yu J, et al. Immunogenicity of COVID-19 mRNA Vaccines in Pregnant and Lactating Women. JAMA. 2021 May 13;

10. Baird JK, Jensen SM, Urba WJ, et al. SARS-CoV-2 antibodies detected in human breast milk post-vaccination. medRxiv. 2021 Mar 10

11. Coronavirus disease (COVID-19): Vaccines safety. Accessed May 15, 2021. Available from: $h t t p s: / / w w w . w h o . i n t / n e w s-r o o m / q-a-d e-$ tail/coronavirus-disease-(COVID-19)-vaccines-safety

12. Vaccinating Pregnant and Lactating Patients Against COVID-19. Accessed May 15, 2021. Available from: https://www.acog.org/en/ clinical/clinical-guidance/practice-advisory/articles/2020/12/ vaccinating-pregnant-and-lactating-patients-against-COVID-19

13. COVID-19 Vaccination for pregnant and breastfeeding women. Accessed May 15, 2021. Available from: https://www.figo.org/ COVID-19-vaccination-pregnant-and-breastfeeding-women

14. FOGSI position statement COVID vaccination for pregnant and breastfeeding women. The Federation of Obstetric and Gynecological Societies of India. Accessed May 15, 2021. Available from: https://www.fogsi.org/COVID-vaccination-for-pregnant-bfwomen/

15. COVID-19 vaccination - Shared decision making guide for women who are pregnant, breastfeeding or planning pregnancy. Australian Government Department of Health. Accessed May 15, 2021. Available from: https://www.health.gov.au/resources/publications/ COVID-19-vaccination-shared-decision-making-guide-forwomen-who-are-pregnant-breastfeeding-or-planning-pregnancy

16. COVID-19 vaccines in pregnancy and breastfeeding. The immunization advisory centre. Accessed May 15, 2021. Available from: https://COVID.immune.org.nz/faq/COVID-19-vaccines-pregnancy-and-breastfeeding

17. Davanzo R, Agosti M, Cetin I, et al. Breastfeeding and COVID-19 vaccination: Position statement of the Italian scientific societies. Ital J Pediatr. 2021;47:45. 